\title{
PROPUESTA PARA LA RECOLECCIÓN DE MICROVESTIGIOS ARQUEOBOTÁNICOS EN MORTEROS FIJOS
}

\section{A PROPOSAL FOR THE RECOVERY OF ARCHAEOBOTANICAL MI- CROREMAINS IN NON-MOBILE MORTARS}

\author{
Marco Giovannetti \\ CONICET-División Arqueología, Museo de Ciencias Naturales FCNyM, UNLP. \\ E-mail: mgiovannetti@conicet.gov.ar
}

Presentado el: 10/12/2012 - Aceptado 24/02/2013

\section{Introducción}

La investigación de microvestigios vegetales, también conocidos como microrrestos, ha evolucionado con rapidez en los últimos años de la práctica arqueológica en general. Como parte de lo que se conoce como especialización arqueobotánica el estudio de los granos de almidón, fitolitos, fibras celulares y algunos otros elementos orgánicos de las plantas han sido interpelados por la arqueología dando buenos resultados en diversos tipos de problemas (Coil et al. 2003). Estos vestigios arqueológicos han sido recuperados desde distintos tipos de objetos y/o espacios que se hipotetizaba -a veces con sólidas bases inferenciales y otras no tanto- estuvieron en contacto directo con vegetales. Es el caso de objetos arqueológicos líticos como hachas, raspadores e instrumentos de molienda, tanto el componente activo (mano de moler) como pasivo (la cavidad que posee varios nombres dependiendo de los tipos) sobre los que se comprobó la eficacia de esta técnica ${ }^{1}$. Recientemente también se está comenzando a explorar el potencial de hallazgo sobre piezas cerámicas y piezas dentales humanas (Zucol et al 2008, Lantos et al 2012, Musoubach 2012). En esta presentación nos interesa brindar un método para una correcta y productiva recolección de muestras con posibles microrrestos vegetales en un tipo de restos arqueológicos particulares como es el caso de los morteros fijos, artefactos constituidos sobre grandes bloques inmóviles, muy comunes en algunas regiones de nuestro país como el Noroeste Argentino (NOA) y Sierras Centrales de Argentina. La especificidad de este tipo de instrumento de molienda ha sido muy poco estudiada siendo en general tratados sin independencia de los objetos móviles. En determinados trabajos (Nelson y Lippmeier 1993) es posible consignarlos y analizarlos como parte del moviliario fijo y duradero de sitios arqueológicos (site fourniture) y por ende pasibles de reutilización. Exactamente desde aquí nace la necesidad de particularizarlos 
a partir de sus propias características y potencialidades, es decir, dado que son objetos inmóviles que deben ser trabajados en el campo, requieren de una metodología propia para la extracción de restos analizables en laboratorio. Los morteros móviles, en cambio, pueden ser transportados al gabinete de trabajo y allí mismo extraer las muestras para recuperar, describir e identificar los microrrestos vegetales.

A partir de una investigación concreta del sitio El Shincal de Quimivil (Catamarca) -donde la cantidad de rocas inmóviles con morteros en su superficie fue muy abundante tanto en el sitio como en zonas cercanas- fue necesario focalizar sobre los mismos caracterizándolos y articulándolos con la dinámica del sitio Inka (Giovannetti 2009). Se trabajó a nivel de conjunto de molienda o mortero múltiple cuando se trataba de rocas inmóviles que contenían dos o más unidades de molienda (cada una de las cavidades de molienda) discriminando a su vez por distintas morfologías fácilmente discernibles. Aunque pondremos este caso como ejemplo en esta presentación queremos apuntar nuestro objetivo sobre la metodología utilizada para la posterior identificación de microrrestos vegetales provenientes de los morteros. Sobre algunos ejemplares se realizó la extracción de sedimento inmediato a las paredes de las unidades de molienda arrojando, luego de los análisis de laboratorio, excelentes resultados.

Sintetizando, mostraremos algunas formas útiles de proceder en el campo para extraer muestras de sedimentos factibles de contener microrrestos vegetales desde instrumentos como los morteros múltiples, que consecuentemente resultan imposibles de movilizar desde su sitio original.

\section{Propuesta Metodológica}

\section{Extracción de sedimento en seco}

Una forma sumamente eficaz para conseguir muestras es el raspado profundo e intenso de las paredes al interior de la oquedad de molienda. En primera instancia se debe llegar a tener contacto con las mismas, es decir, poco sirve para el reconocimiento de recursos procesados el sedimento de relleno que en muchas ocasiones ha colmatado completamente la cavidad de molienda dado que se trata de elementos casi con seguridad postdeposicionales ${ }^{2}$. En algunas ocasiones este problema no se presenta, encontrándose las cavidades de molienda descubiertas al aire libre. En este caso particular deberá asegurarse bien que el mortero arqueológico no haya sido reutilizado en tiempos recientes por pobladores locales lo que alteraría completamente la evidencia de molienda de momentos previos. Pero en cualquiera de las formas en que pueda hacerse contacto con las paredes y base de las cavidades es factible obtener resultados exitosos.

Procedimiento: en primera instancia se tomará una muestra del sedimento que rellena la oquedad a fin de ser utilizada como muestra testigo. Una vez realizado esto y extraído el relleno posdeposicional, se deberá acumular sedimento proveniente de las paredes y la base de la oquedad a fin de ser colectado y guardado adecuadamente. Para ello se rasparán y pincelarán estos sectores removiendo el sedimento adherido sobre los mismos. No debe desalentar la escasez de material colectado. Aún cuando las chances de recuperar microrrestos son más altas cuanto más sedimento pueda recolectarse de estos sectores, desde experiencias personales podemos asegurar que con algunos miligramos del mismo pueden reconocerse elementos significativos. Este material debe ineludiblemente recogerse y almacenarse adecuadamente para su traslado. El sedimento no necesariamente debe 
trasladarse de inmediato al laboratorio de análisis, no existe peligro de degradación bajo condiciones de almacenamiento adecuadas. Se debe colocar el sedimento dentro de una primera bolsa o tubo de plástico estéril (ver abajo) sin agregar etiqueta o rótulo dentro para evitar contaminación. Este último debe añadirse en una segunda bolsa del mismo material para no perder las referencias pertinentes. Es necesario poseer un plano o esquema de las diferentes unidades de molienda con previa nominación o numeración de cada una. De esta forma se tendrá referencia adecuada al momento de extraer y etiquetar.

Instrumental: el barrido de las paredes y base de la oquedad de molienda puede realizarse con un pincel pequeño o mediano de cerda fina regulando la intensidad dependiendo de cuanto pueda recolectarse. Los elementos de metal o plástico son los más idóneos para recoger lo que haya sido acumulado mediante el barrido. Cucharas finas y elongadas resultan adecuadas para las cavidades profundas ${ }^{3}$ (Figura 1a). Cucharas más anchas y cortas son útiles para cavidades poco profundas y anchas como la de las conanas (Figura 1b). Cualquier otra cuchara de metal de dimensiones adaptables a los instrumentos de molienda arqueológicos puede ser también adecuada.

El sedimento puede almacenarse en cualquier contenedor de polietileno (plástico) ya sea a manera de bolsa o tubo pequeño con tapa firme como aquellos de rollos de fotografía. En el caso de las bolsas las más adecuadas son aquellas que están equipadas con cierre a presión hermético.

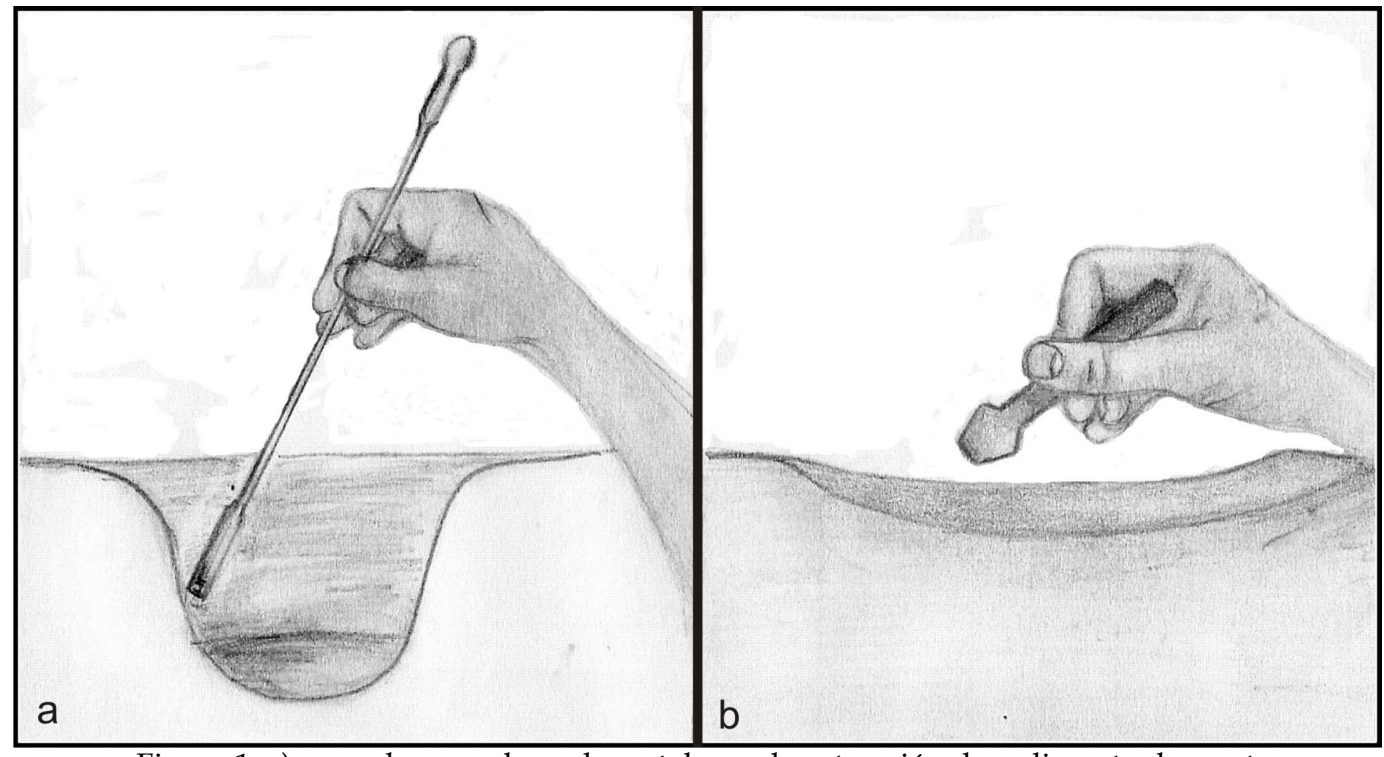

Figura 1. a) raspador y cuchara de metal para la extracción de sedimento de morteros profundos. b) Cuchara de metal corta y ancha para raspado y recolección de sedimento de morteros poco profundos.

Precauciones: uno de los problemas principales del trabajo con restos de almidón arqueológicos es el peligro de contaminación con almidones provenientes de cualquier otra fuente, en general modernos (Wadley y Lombard 2006). Es por ello que son varios los resguardos que se deben tomar. En primer lugar impedir cualquier contacto, e incluso cercanía, de alimentos, sobre todo aquellos de naturaleza almidonosa (pan, galletas, etc.). Los almidones 
que contienen no solo pueden caer sobre los restos arqueológicos sin que siquiera pueda notarse sino que el viento puede trasladarlos hacia allí si se los está manipulando en las cercanías. También las manos mismas son un foco peligroso de contaminación si no se han limpiado y lavado cuidadosamente antes de llevar adelante la extracción de las muestras de sedimento. Partículas muy pequeñas de harinas pueden quedar adheridas a las manos luego de consumir alimentos y estos pueden permanecer por largo tiempo. Por ello es fundamental un minucioso proceso de lavado y secado previo. No se recomienda en lo absoluto la utilización de guantes de látex pues los mismos suelen ser entalcados con fécula de maíz y por ende harían peligrar la muestra arqueológica (Wadley et al. 2004). Pueden en cambio usarse guantes estériles. Las mismas precauciones debemos tomar en relación a los instrumentos a utilizar, hay que mantenerlos lejos de cualquier fuente de contaminación ${ }^{4}$ así como limpiarlos minuciosamente antes de cada extracción.

Para manipular los recipientes que contienen las muestras de sedimento recolectadas se exige el mismo cuidado que para la recolección, incluso en el espacio del laboratorio de análisis. Pero debe tenerse en cuenta otra situación para evitar contaminación, esta vez de otras cavidades de molienda. Suele darse el caso de conjuntos de molienda con varias unidades en su superficie. Si se desea extraer muestras de más de una unidad debe ponerse atención sobre los instrumentos de recolección. Primeramente nunca deben mezclarse los sedimentos de una y otra unidad arqueológica. Luego, debe tomarse particular cuidado en relación a partículas que pudieran quedar adheridas al instrumental producto de la recogida previa. Para ello debe limpiarse muy bien el pincel usado o utilizar uno nuevo, al igual que las otras herramientas.

\section{Extracción líquida}

Puede ocurrir el caso del hallazgo de conjuntos de molienda no enterrados, es decir completamente al aire libre, y con nula depositación de sedimento en su interior. Si el barrido de la cavidad interior resulta infructuoso entonces podría utilizarse la técnica de extracción líquida para recuperar almidones $\mathrm{u}$ otros restos atrapados en las cavidades minúsculas del interior de la unidad de molienda.

Procedimiento: debe volcarse dentro de la cavidad de molienda agua destilada en una cantidad que luego pueda ser transportada en recipientes manipulables $(100 \mathrm{ml}$ puede ser una cantidad recomendable). Con un instrumento de metal o plástico como los señalados arriba se puede agitar el medio líquido tratando de producir roce con la superficie rocosa. De esta manera se facilita la liberación de partículas arqueológicas adheridas. Se extrae la mayor cantidad posible del líquido con residuos de la unidad de molienda y se puede proceder de dos formas diferentes para su almacenamiento y transporte:

1- se puede depositar el material líquido en un frasco o tubo con una tapa de cierre fuerte y sólida. Tomar todos los resguardos necesarios para evitar pérdida del mismo sobre todo a nivel de la tapa señalada. Para este caso, por ejemplo, se puede poner cinta selladora. Este procedimiento posee la ventaja de evitar múltiples trasvases del líquido, ya que en laboratorio se puede extraer la muestra directamente para procesar y observar. La desventaja transcurre en el peligro de perdida de material ante la inestabilidad del medio líquido para permanecer en su envase sobre todo contemplando el movimiento de traslado. 
2-Según Babot $(2004$, 2007) el líquido extraído de la cavidad de molienda puede colocarse en viales para su posterior evaporación. Luego se utilizarían pequeñas cantidades de alcohol para montar los residuos deshidratados. Remitimos al trabajo de la autora citada donde brevemente explica este método en caso de necesidad de replicarlo.

En ambos casos es necesario rotular los recipientes transportadores preferentemente con marcador indeleble sobre alguno de sus laterales.

Instrumental: las pipetas Pasteur y las jeringas de presión son las mejores herramientas para volcar y extraer el líquido de las oquedades de molienda. Las cucharas largas de metal o plástico -similares a las de la técnica en seco- son las que se usarían para agitar el líquido volcado sobre la cavidad de molienda. Para el caso del procedimiento 1 de envasado cualquier frasco de vidrio o plástico con fuerte cierre hermético podrá evitar el derramamiento del líquido en el transporte posterior. En el procedimiento 2 serían necesarios viales de vidrio que permitirán contener el líquido mientras la muestra se deshidrata.

Precauciones: Debe garantizarse la correcta limpieza y manipulación de todo este material, tanto el instrumental como el arqueológico, para evitar contaminación de la misma forma que describimos en el caso de la extracción de sedimento en seco. Luego es necesario poner mucho cuidado en evitar la pérdida de muestra líquida en el trasvase de un recipiente a otro o en el transporte de los tubos almacenadores. Para ello se requiere de fuertes tapas a rosca o a presión. Para esto último se recomienda posicionar siempre el contenedor verticalmente con la tapa hacia arriba, evitando su colocación en cualquier tipo de bolsa. En el caso del procedimiento 2 debe evitarse evaporar el líquido aplicando calor ya que los almidones son altamente sensibles a los aumentos de temperatura provocando la desnaturalización de los mismos.

\section{Caso de estudio. Un ejemplo a partir de los morteros de El Shincal de Quimivil}

La primera recolección de microvestigios vegetales sobre un mortero múltiple en nuestro sitio de estudio se realizó sobre uno de los ejemplares más llamativos por su tamaño y cantidad de unidades de molienda, el mortero múltiple EGP (Figuras 2 y 3). La gran roca contiene 61 unidades de molienda en su superficie totalmente pulida tanto en las oquedades como en los espacios entre ellas. Se ubica a poco más de $1 \mathrm{~km}$ del núcleo de las ruinas inka y según nuestros estudios se habría articulado con la dinámica del sitio a partir de la producción de chicha a gran escala. Según los resultados de las excavaciones, la molienda y los primeros momentos de cocción de la chicha se producía en las inmediaciones del mortero (para mayor desarrollo ver Giovannetti 2009, Giovannetti et al. 2010).

Se seleccionó para la recuperación de muestras de sedimento el método de extracción en seco tal como lo explicáramos arriba. Se utilizó como instrumental para el raspado y removido de sedimento de las paredes de las unidades de molienda una cuchara de acero inoxidable de $20 \mathrm{~cm}$ de longitud y $0,6 \mathrm{~cm}$ de espesor en su extremo. Con la misma y con ayuda de un pincel de cerda fina se extrajo el sedimento que fue resguardado en doble bolsa de sellado hermético. La etiqueta con los datos de procedencia fue colocada en la segunda bolsa. Se recogió sedimento de 21 unidades de molienda tanto de las paredes como del relleno para aplicar como casos de control. Se seleccionó sólo unidades que 


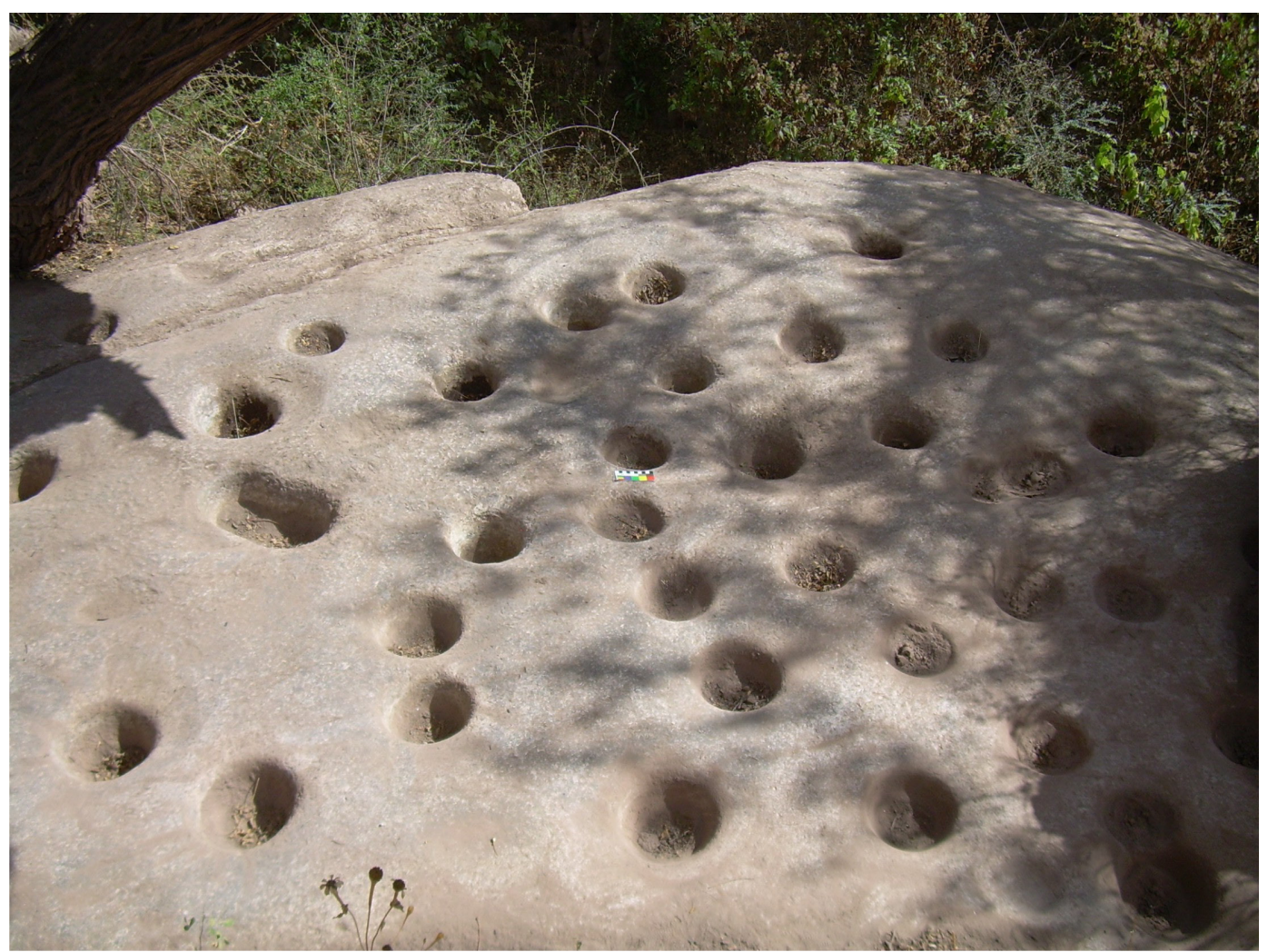

Figura 2. Mortero múltiple, conjunto EGP

se encontraran lo suficientemente cubiertas de sedimento que permitiera inferir que no fueron utilizados en mucho tiempo, es decir con prácticamente nulas probabilidades de reutilización reciente. El instrumental en todos los casos fue cuidadosamente limpiado luego de la extracción de cada uno de los morteros.

Una vez en el laboratorio nos enfrentamos al problema ya conocido del tamaño de los granos de sedimento que solaparían la localización de cualquier microvestigio arqueológico en la observación microscópica (Babot 2007). Ante esto decidimos tamizar el material a observar sobre una malla especial de $90 \mu$, una medida que consideramos razonable para dejar pasar los microvestigios arqueológicos que por lo general tienen tamaños por debajo de esta medida.

Para la observación a altos aumentos fue utilizado un microscopio de luz reflejada y transmitida (Leica DM/LM) provisto de equipo de cámara clara, luz polarizada y cámara fotográfica digital incorporada. Las muestras de sedimento a observar fueron colocadas en portaobjetos, manipuladas con instrumental de metal lavado y expuesto al fuego luego de cada sesión con una muestra. A la muestra de sedimento sobre el portaobjetos se le sumó líquido de montaje compuesto de glicerina al 50\%, una medida confiable que evita la desecación, sumado a que su relativa viscosidad permite una adecuada manipulación para obtener imágenes en distintos ángulos de los cuerpos observados. Además aumenta la birrefringencia del preparado (Pagán Jiménez et al. 2005). El agua utilizada para preparar la muestra siempre fue destilada. Los porta y cubreobjetos utilizados nunca tuvieron utilización previa en otra observación y fueron directamente extraídos del envase original. La mera 
y simple manipulación de laboratorio puede alterar notablemente y de múltiples maneras una muestra para analizar (Coil et al. 2003). Por ello siempre se trabajó a mano desnuda pero con especial cuidado en su lavado consecutivo ya que, según nuestra experiencia, son uno de los factores contaminantes más importantes, sobre todo de almidones provenientes de harina de trigo.

Procedimos a analizar tres portaobjetos para cada unidad de molienda con una cantidad adecuada de sedimento que no colmara el campo visual al punto de volverse imposible localizar microvestigios arqueológicos. Trabajamos con cantidades fijas de sedimento de $50 \mathrm{mg}$. para todas las unidades de molienda. Esta magnitud pequeña y un tanto difícil de manipular fue medida con una balanza de precisión electrónica. La cantidad de muestra colocada en cada portaobjetos fue observada completamente siguiendo un procedimiento ordenado.

Los resultados obtenidos se resumen en la tabla 1. Como se puede observar el número de ejemplares de almidón recuperados fue significativo. Sin ahondar en el análisis de los mismos, dado que solo se pretende mostrarlos a manera de ejemplo de esta aplicación metodológica, se puede apreciar que algunas unidades de molienda no arrojaron resultados mientras que otras sí en números importantes (unidades 4 u 11 por ejemplo). En relación a los gránulos de almidón específicamente hubo ejemplares con buen estado de conservación que pudieron ser muy bien identificados taxonómicamente. Otros en cambio presentaban algún grado de alteración (en algunos casos propio del machacado en el mortero) y pudieron ser relacionados con alguna especie botánica aunque con cierta reserva (el caso de los clasificados como "posibles").

Para contrastar la correlación de estos hallazgos con residuos de prácticas de molienda analizamos el sedimento que rellenaba los morteros (Tabla 1 "sedimento testigo"). Sólo algunas unidades presentaron restos reconocibles de gránulos de almidón pero todos resultaron ser ejemplares primarios, es decir aquellos que la planta no utiliza para almacenar nutrientes y por ende no tienen potencialidad taxonómica para ser identificados.

\begin{tabular}{|c|c|c|c|c|c|c|c|c|c|c|c|c|c|c|c|}
\hline $\begin{array}{l}\text { Unidades } \\
\text { EGP }\end{array}$ & \begin{tabular}{|l} 
mays \\
mays
\end{tabular} & \begin{tabular}{|l|} 
Posible \\
$Z$ mays \\
\end{tabular} & $\begin{array}{l}\text { Indet. Afin } \\
\text { Z. mays }\end{array}$ & $\begin{array}{l}\begin{array}{l}\text { Phaseolus } \\
\text { vulgaris }\end{array} \\
\end{array}$ & \begin{tabular}{|l|} 
Posible \\
Phaseolus \\
vulgaris
\end{tabular} & $\begin{array}{l}\text { Phase olus o } \\
\text { Triticur }\end{array}$ & Triticum & \begin{tabular}{|l|} 
Tubérculo \\
andino
\end{tabular} & \begin{tabular}{|l|l}
$\begin{array}{l}\text { Posible } \\
\text { tubérculo } \\
\text { andino }\end{array}$ \\
\end{tabular} & \begin{tabular}{|l|}
$\begin{array}{l}\text { Indet. Afin } \\
\text { tubérculo } \\
\text { andino }\end{array}$ \\
\end{tabular} & $\begin{array}{l}\text { Prosopi } \\
\text { s }\end{array}$ & \begin{tabular}{|l} 
Posible \\
Prosopis
\end{tabular} & Indet. & Total & \begin{tabular}{|l}
$\begin{array}{l}\text { Sedimento } \\
\text { testigo }\end{array}$ \\
\end{tabular} \\
\hline Unidad 4 & 6 & & 1 & 1 & 2 & 2 & & & & & & 1 & 7 & 23 & 0 \\
\hline Unidad 11 & 6 & & & & 1 & & & & & & & & 3 & 21 & $\begin{array}{l}1 \text { (almidón } \\
\text { primario) }\end{array}$ \\
\hline \begin{tabular}{|l|l} 
Unidad 14 \\
\end{tabular} & 3 & & & 2 & & 3 & & & & & & & 2 & 11 & 0 \\
\hline Unidad 19 & 5 & & & 3 & & 2 & & & & & & & 1 & 12 & 0 \\
\hline \begin{tabular}{|l} 
Unidad 2 \\
\end{tabular} & & 2 & & & & & & & & & & & 7 & 2 & 0 \\
\hline \begin{tabular}{|l|l} 
Unidad 25 \\
\end{tabular} & 7 & & & & & & & & 1 & & & & 1 & 14 & 0 \\
\hline \begin{tabular}{|l|l} 
Unidad 31 \\
\end{tabular} & & & & & & & & & & & & & 3 & 5 & 0 \\
\hline Unidad 35 & 3 & & & 2 & 1 & & & & & & & & & & $\begin{array}{l}2 \text { (almidón } \\
\text { primario) }\end{array}$ \\
\hline \begin{tabular}{|l|l} 
Unidad 36 \\
\end{tabular} & 2 & & & 2 & 2 & & & & & & & & 2 & 8 & 0 \\
\hline Unidad 37 & 2 & & & & & & & & 1 & & & & 1 & 4 & 0 \\
\hline Unidad 38 & & & & & & & & & & & & & & 5 & 0 \\
\hline Unidad 41 & & & & & & & & & & & & & & 0 & 0 \\
\hline Unidad 42 & 2 & & & & & 1 & & & & & & & 2 & 5 & 0 \\
\hline Unidad 46 & & & & & & & & & & & & & & & $\begin{array}{l}1 \text { (almidón } \\
\text { primario) }\end{array}$ \\
\hline Unidad 47 & 2 & & & & 1 & & & & & & & & & 3 & 0 \\
\hline \begin{tabular}{|l|l|} 
Unidad 48 \\
\end{tabular} & & & & & & & & & & & & & & 0 & 0 \\
\hline Unidad 50 & 1 & & & & & & & & & & 1 & & & 2 & 0 \\
\hline Unidad 58 & 1 & & & 2 & & 2 & & 1 & & & & & 2 & 10 & 0 \\
\hline Unidad 59 & & & & & & & & & 1 & & & & & & $\begin{array}{l}1 \text { (almidón } \\
\text { primario) }\end{array}$ \\
\hline Unidad 60 & & & & & & & & & & & 1 & & & 1 & 0 \\
\hline Unidad 61 & 1 & & & & & & & & & & & & & 1 & 0 \\
\hline TOTAL & 41 & 15 & 1 & 13 & 7 & 16 & & 6 & 3 & & 6 & 2 & 24 & 139 & 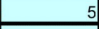 \\
\hline$\%$ & 29,5 & 10,79 & 0,72 & 9,35 & 5,04 & 11,51 & 2,88 & 4,32 & 2,16 & 0,72 & 4,32 & 1,44 & \begin{tabular}{|l|}
17,27 \\
\end{tabular} & 100 & \\
\hline
\end{tabular}

Tabla 1. Gránulos de almidón identificados en los sedimentos extraídos del mortero EGP 

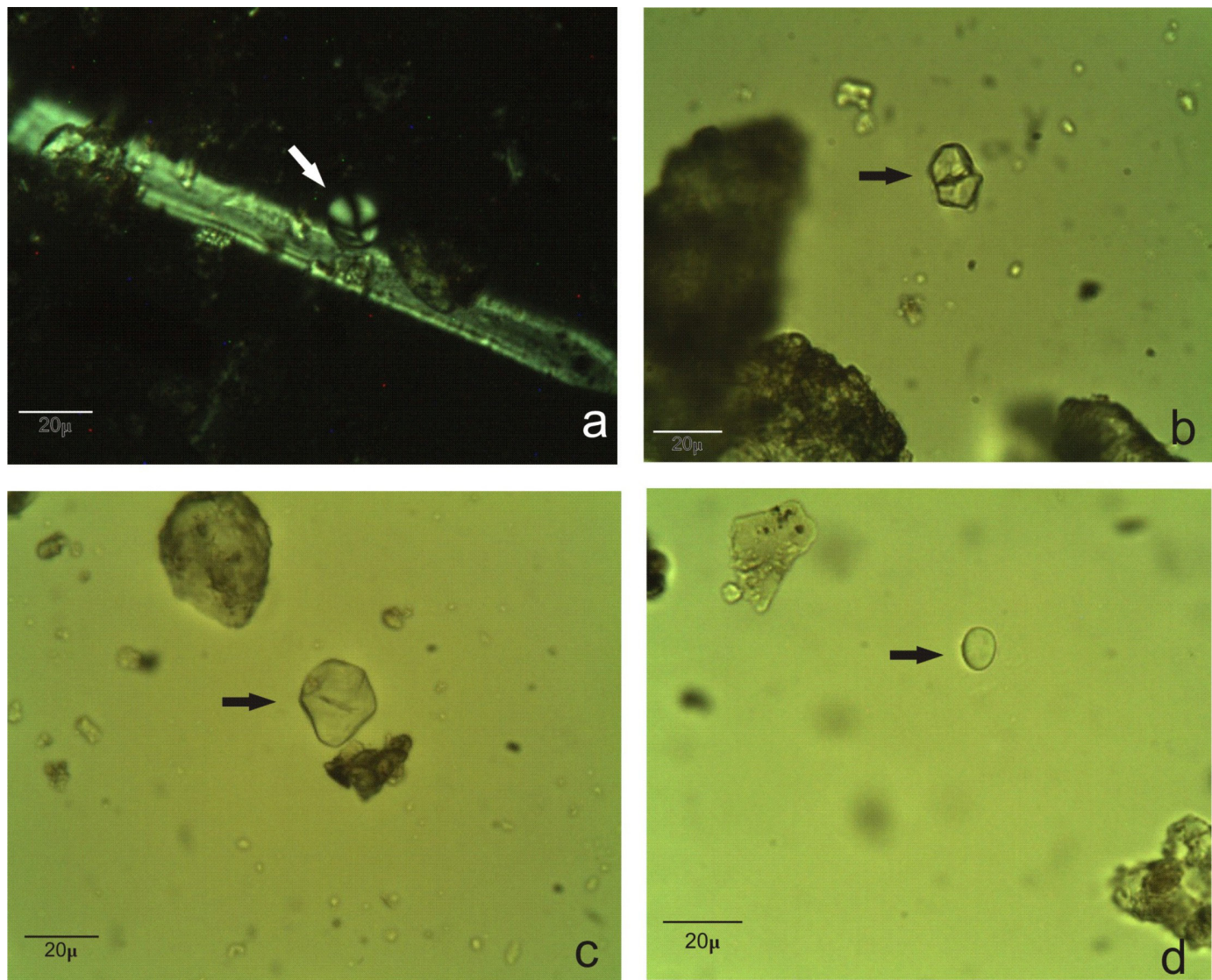

Figura 3. Ejemplares de gránulos de almidón obtenidos del mortero EGP. a) Tubérculo andino, afín S. tuberosum observado bajo luz polarizada. b) Prosopis sp. c) Z. mays endosperma blando. d) Phaseolus vulgaris

Estos resultados permitieron un grado confiable de aceptación para inferir que los gránulos de almidón de las paredes de los morteros se corresponden con productos molidos al momento en que el sitio arqueológico se encontraba en pleno desarrollo, tanto en tiempos prehispánicos como inmediatamente posteriores (períodos Inka e Hispano-indígena).

\section{Comentarios Finales}

Es necesario en las investigaciones arqueológicas sudamericanas aumentar los estudios sobre los artefactos de molienda, especialmente aquellos que han recibido escaso tratamiento como los morteros múltiples fijos. El potencial de los estudios microscópicos en arqueobotánica es muy grande para este tipo de instrumentos fijos, tal como lo han demostrado algunas investigaciones realizadas en el NOA recientemente (Babot y Apella 2003; Giovannetti et al. 2008; Giovannetti 2009 entre otros). Aún así es imprescindible focalizar ordenada y sistemáticamente las investigaciones sobre estos vestigios arqueológicos para contrastar muchos supuestos escasamente argumentados tanto sobre funcionalidad como contextualización cultural y temporal (ver para esta discusión Babot 2008, 2009; Giovannetti 2009). Pero además, al ser objetos altamente visibles, resistentes y numerosos (evidencia material de su importancia en la vida de las sociedades del pasado americano), son depositarios de mucha información fundamental para la arqueología. Por 
ejemplo, es posible conocer productos molidos y relacionarlos con comidas y bebidas y las maneras articuladas culturalmente para cada caso. También la socialización del trabajo de molienda y su inserción en las prácticas y organización del proceso de producción. Por otra parte también pueden estudiarse las técnicas de fabricación de estos medios de producción. Para ello no sólo se requieren sólidos análisis de vestigios microscópicos sino también un adecuado registro morfométrico y caracterizaciones físicas detalladas.

En el caso de nuestros estudios en El Shincal de Quimivil pudimos relacionar las prácticas de trabajo en los morteros múltiples y las festividades oficiadas y auspiciadas desde el Estado Inka. Pudimos constatar certeramente que dentro de los morteros se estaba machacando maíz, poroto, algarroba e incluso tubérculos cultivados. En los primeros tres casos pudimos corroborar la presencia de los mismos a través de macrorestos carbonizados pero en el caso de los tubérculos fue toda una novedad encontrar los almidones dado que no se preservó de otra manera. Otro aporte muy importante fue la identificación de trigo. Es destacable su presencia dado que nos aporta un marcador temporal que complementa otros hallazgos dentro de los macrorestos como granos de cebada y semillas de uva. Sabemos que El Shincal tuvo un evento de ocupación relativo al período Hispano-indígena. Con seguridad estos restos se corresponderían con esto indicándonos la utilidad de los grandes morteros múltiples a lo largo de los siglos XV y XVI según nuestros fechados radiocarbónicos (Giovannetti 2009).

La propuesta aquí presentada no intenta convertirse en el manual de procedimiento absoluto, sino una guía para la recolección de un tipo de evidencia delicada y fácilmente destruible por la manipulación humana, ya sea tanto de arqueólogos como de personas que los reclaman desde el contexto arqueológico al contexto sistémico. Pero apoyándonos en una criteriosa y sistemática recolección de sedimentos de su interior, aún sin un análisis inmediato de las muestras recogidas, es posible resguardarlos por mucho tiempo hasta su análisis ulterior. Es en este sentido que esta presentación intenta colaborar con una práctica arqueológica que pueda resguardar, hasta el límite de lo posible, información que en muchos casos se pierde por desconocimiento propio de los arqueólogos.

\section{Agradecimientos}

A Sofía Silva por los dibujos. Al CONICET por el apoyo económico de todos los proyectos encarados desde mi equipo de investigación

\section{Notas}

1- Sólo por poner algunos ejemplos en América de interesantes resultados obtenidos sobre instrumentos de naturaleza pétrea ver Piperno y Holst (1998), Babot y Apella (2003), Babot (2004), Pearsall et al (2004), Perry (2004), Capdepont et al (2005), Zucol y Bonomo (2008).

${ }^{2-}$ El sedimento que colmata una cavidad de molienda puede utilizarse, sin embargo, como testigo para la comparación con el sedimento extraído de las paredes del interior de los morteros (ver Capdepont et al 2005 para el caso de morteros móviles).

3- En tiendas de instrumental quirúrgico u odontológico se encontrarán herramientas de metal muy idóneas por su resistencia y durabilidad. También dentro de los instrumentos utilizados para pintura y cerámica existe una variedad enorme de espátulas y elementos de raspado y corte que resultarían muy adecuados para esta tarea.

${ }^{4-}$ La utilización de herramientas de metal o plástico se relaciona justamente con la precaución de impedir que restos vegetales, en el caso de la madera, se introduzcan desde fuera del contexto arqueológico. 


\section{Bibliografía citada}

Babot, $\mathrm{M}$

2004 Tecnología y utilización de artefactos de molienda en el Noroeste prehispánico. Tesis Doctoral inédita, Facultad de Ciencias Naturales e IML, Universidad Nacional de Tucumán.

2007 Granos de almidón en contextos arqueológicos: posibilidades y perspectivas a partir de casos del Noroeste Argentino. Investigaciones arqueobotánicas en Latinoamérica: estudios de casos y propuestas metodológicas (ed por B. Marconetto, N. Oliszewski, y M. Babot). Pp: 95-125 Centro Editorial de la Facultad de Filosofía y Humanidades - UNC. Córdoba.

2008 Reflexiones sobre el abordaje de la molienda vegetal desde una experiencia de integración disciplinaria. Arqueobotánica y teoría arqueológica. Discusiones desde Sudamérica (comp. por S. Archila, M. Giovannetti y V. Lema V. Pp. 203-230. Uniandes, Bogotá.

2009 Organización social de la práctica de molienda: casos actuales y prehispánicos del Noroeste Argentino. Procesos sociales prehispánicos en el sur andino. La Vivienda, la comunidad y el territorio (ed. por A. Nielsen, C. Rivolta, V. Seldes, M. Vázquez, y P. Mercolli. pp 259290. Editorial Brujas, Córdoba.

Babot, M. y M. Apella

2003 Maize and bone: residues of grinding in Northwestern Argentina. Archaeometry 45 (1): 121-132.

Capdepont I., L. del Puerto y H. Inda

2005 Instrumentos de molienda: evidencias del procesamiento de recursos vegetales en la laguna de Castillos - Rocha, Uruguay. Intersecciones en Antropología 6: 3-19.

Coil, J., M. Korstanje, S. Archer y C. Hastorf

2004 Laboratory goals and considerations for multiple microfossil extraction in archaeology. Journal of Archaeological Science 30: 991-1008.

Giovannetti, M.

2009 Articulación entre el sistema agrícola, redes de irrigación y áreas de molienda como medida del grado de ocupación Inka en El Shincal y Los Colorados (Prov. de Catamarca). Tesis de doctorado inédita. FCNyM, UNLP.

Giovannetti, M., V. Lema, C. Bártoli y A. Capparelli

2008 Starch grains characterization of Prosopis chilensis (Mol.) Stuntz and P. flexuosa DC, and the analysis of archaeological remains in Andean South America. Journal of Archaeological Science 35: 2973-2985.

Giovannetti, M., Cochero, G., Espósito, P. y J. Spina

2010 Excavación y análisis de un mortero múltiple a través de la diversidad de su registro y su relación con la evidencia cerámica. Arqueología Argentina en el Bicentenario de la Revolución de Mayo Publicaciones del XVII Congreso Nacional de Arqueología Argentina. Tomo I (ed por R. Bárcena y H. Chiavazza). Pp: 163- 168 Mendoza.

Lantos, I., M. Maier y N. Ratto

2012. Recreando recetas: una experimentación con variedades nativas de maíz del Noroeste Argentino. Las manos en la masa arqueologías y antropologías de la alimentación en Suramérica (Ed. por M. Babot, M. Marschoff y F. Pazzarelli) Pp. 533-558. Corintios 13, Córdoba. 
Musaubach, M.

2012 Potencialidad de estudios arqueobotánicos sobre tártaro dental de cazadores recolectores de la provincia de La Pampa, Argentina. Revista Argentina de Antropología Biológica Volumen 14, Número Especial Pp: 105-113.

Nelson, M. y H. Lippmeier

1993 Grinding- tool design as conditioned by land-use pattern. American Antiquity 58 (2): 286-305.

Pagán Jiménez, J.; M. Rodríguez López; L. Chanlatte Baik e Y. Narganes Storde 2005 La temprana introducción y uso de algunas plantas domésticas, silvestres y cultivos en Las Antillas precolombinas. Una primera revaloración desde la perspectiva del "arcaico" de Vieques y Puerto Rico. Diálogo Antropológico 3 nro. 10. Pp: 7-33.

Pearsall, D., K. Chandler-Ezellb y J. Zeidlerc

2004 Maize in ancient Ecuador: results of residue analysis of stone tools from the Real Alto site. Journal of Archaeological Science 31: 423-442.

Perry, L.

2004 Starch analyses reveal the relationship between tool type and function: an example from the Orinoco valley of Venezuela. Journal of Archaeological Science 31: 1069-1081.

Piperno, D. e I. Holst

1998 The Presence of Starch Grains on Prehistoric Stone Tools from the Humid Neotropics: Indications of Early Tuber Use and Agriculture in Panama. Journal of Archaeological Science 25: 765-776.

Wadley, L.; M. Lombard y B. Williamson

2004 The first residue analysis blind tests: results and lessons learnt. Journal of Archaeological Science 31:1491-1501.

Wadley, L. y M. Lombard

2006 Small things in perspective: the contribution of our blind tests to micro-residue studies on archaeological stone tools. Journal of Archaeological Science 34: 1001-1010.

Zucol, A. y M. Bonomo

2008 Estudios etnobotánicos del sitio arqueológico Nutria Mansa 1 (partido de General Alvarado, provincia de Buenos Aires): II. Análisis fitolíticos comparativos de artefactos de molienda. Matices interdisciplinarios en estudios fitolíticos y de otros microfósiles (ed. por A. Korstanje y M. Babot) Pp: 173-185. BAR International Series, Oxford.

Zucol, A., M. Brea y D. Mazzanti

2008 Análisis de restos orgánicos presentes en cerámicas arqueológicas de las sierras de Tandilia (provincia de Buenos Aires, Argentina). Matices Interdisciplinarios en Estudios Fitolíticos y de Otros Microfósiles (ed. por M. A. Korstanje y M. Babot). Pp. 201-208. BAR International Series 